\title{
Ocupación de cajas-refugio por murciélagos en Navarra
}

\author{
Juan Tomás Alcalde*, David Campion, Javier Fabo, Felipe Marín, \\ Alberto Artázcoz, IÑAKi Martínez, InMaCUlada Antón
}

Plaza Sabicas, $\mathrm{n}^{\circ} 5,2^{\circ}-31015$, Pamplona (Spain)

* Correo electrónico del autor: jtalcalde@gmail.com

DOI: http://dx.doi.org/10.14709/BarbJ.6.1.2013.05

English title: Occupancy of bat-boxes in Navarre

\begin{abstract}
Navarre (Northern Spain) in autumn 2012. Occupation has ranged between 0 and 90\% depending on the area. Overall, $241(60 \%)$ of the cases showed signs of occupation: 107 (26\%) were inhabited by bats and $134(33 \%)$ contained guano. 345 bats belonging to 10 species have been counted: Myotis mystacinus, Myotis daubentonii, Pipistrellus pipistrellus, Pipistrellus pygmaeus, Pipistrellus kuhlii, Nyctalus leisleri, Nyctalus noctula, Nyctalus lasiopterus, Barbastella barbastellus and Plecotus auritus. The most frequent and abundant were $P$. pygmaeus, found in 53 boxes (247 individuals) and N. leisleri in 24 (36 ind.). Dominant species were found in different areas and species preferences were observed for certain models of boxes. The most used models were $1 \mathrm{FF}$ and $2 \mathrm{~F}$ with double front panel (Schwegler). No significant differences were found in relation to tree species where the boxes are, although there were significant differences in relation to forest structure: the occupation was greater with the more open forest structure. Besides, significant differences were found in relation to the orientation: the largest amount of bats was found in boxes oriented NW, S and SW. $30 \%$ of bat boxes contained other animals and/or obstacles to the bat occupation, mainly excess of guano $(12 \%)$, nests $(9 \%)$ and wasps $(5 \%)$, so regular cleaning is required.
\end{abstract}

Keywords: bat box, roost, conservation, Navarre.

Resumen: En otoño de 2012 se han revisado 405 cajas-refugio para murciélagos, de 7 modelos diferentes en 11 zonas de Navarra (Norte de España). La ocupación ha oscilado entre el 0 y el 90\% según zonas. En conjunto, 241 (60\%) cajas mostraba signos de ocupación: 107 (26\%) estaban habitadas por murciélagos y otras 134 (33\%) contenían guano. Se han contabilizado 345 murciélagos pertenecientes a 10 especies: Myotis mystacinus, Myotis daubentonii, Pipistrellus pipistrellus, Pipistrellus pygmaeus, Pipistrellus kuhlii, Nyctalus leisleri, Nyctalus noctula, Nyctalus lasiopterus, Barbastella barbastellus y Plecotus auritus. Las más frecuentes y abundantes han sido P. pygmaeus, hallada en 53 cajas (247 individuos) y $N$. leisleri, en 24 (36 ind.). Se han encontrado especies dominantes en diferentes zonas y también preferencias de algunas especies por determinados modelos de cajas. Los modelos más utilizados han sido el 1FF y el 2F de doble pared (Schwegler). No se han encontrado diferencias significativas de ocupación en relación a la especie de árbol donde se hallan las cajas, aunque sí respecto a la estructura del bosque: la ocupación es mayor cuanto más abierta es la estructura del bosque. Además se observan diferencias significativas en relación a la orientación: el mayor número de murciélagos se encuentra en las cajas orientadas al NO, S y SO. El 30\% de las cajas presentaban otros animales y/o obstáculos para la ocupación de murciélagos, principalmente exceso de guano (12\%), nidos $(9 \%)$ y avispas $(5 \%)$, por lo que se requiere una limpieza periódica.

Palabras clave: caja, refugio, conservación, Navarra. 


\section{INTRODUCCIÓN}

Navarra se encuentra en una zona de transición bioclimática: en ella confluyen las regiones bioclimáticas alpina, atlántica y mediterránea. Además, su situación geográfica en el norte de la península Ibérica incluye las estribaciones occidentales del Pirineo (Fig. 1), lo que favorece que esta región constituya un territorio de paso para numerosas poblaciones de aves y murciélagos que migran desde Centro Europa hacia el interior de la península y/o África (e.g., Alerstam 1990, Elphick 1995, Ohlendorf et al. 2000, Alcalde et al. en prensa)

En 1998 se colocaron 20 cajas-refugio de madera (Stebbings \& Walsh 1991) en parques de Pamplona (Navarra). Estas cajas pretendían compensar la pérdida casi total de refugios naturales debida a la progresiva tala de prácticamente todos los árboles viejos de la ciudad, ofreciendo refugios alternativos a los nóctulos medianos (Nyctalus noctula) que habitan en los parques de Pamplona. En los tres años siguientes sólo se observó una caja ocupada por nóctulos y además en ese mismo período la mayor parte de las cajas resultaron dañadas por las inclemencias meteorológicas, apreciándose grietas en las juntas y abombamientos de la madera. En verano de 2001 se colocaron 71 nuevas cajas del modelo 2FN de la casa Schwegler (hecha de cemento con fibras de madera en su interior), que fueron rápidamente ocupadas por nóctulos (Alcalde 2008).

El éxito de estas cajas, además de la consideración de que prácticamente todos los bosques de la región son explotados o lo han sido recientemente, y por lo tanto son escasos en oquedades naturales disponibles para murciélagos, motivaron una actitud positiva por parte de los técnicos de la Administración regional encargados de la gestión forestal. Ello desembocó en el desarrollo de una normativa forestal autonómica renovada anualmente (cuya ultima versión es la Orden Foral 118/2012) que incluye como "medida Red Natura 2000" la colocación de cajas-refugio de buena calidad, cuyo coste es sufragado por el Gobierno de Navarra. Esta norma se ha aplicado en la realización de trabajos de gestión forestal $\mathrm{y}$ en proyectos de conservación de la diversidad, siempre que las cajas se encuentren en espacios de la Red Natura 2000.
Las cajas han sido colocadas entre los años 2001 y 2011, por diversos colectivos: técnicos del Gobierno de Navarra, empresas de gestión forestal, organizaciones conservacionistas o particulares. La mayor parte de los proyectos contaron con la participación de expertos en el estudio y conservación de murciélagos; sólo unas pocas fueron colocadas por personas desconocedoras de este grupo animal. Debido a la diversidad de colectivos implicados, se desconoce el número exacto de cajas colocadas en toda la Comunidad, aunque se calcula en torno a 500. Las áreas y hábitats en los que estas cajas han sido colocadas son muy diversos, pero pueden resumirse en:

- Norte de Navarra: grandes masas forestales (hayedos y robledales) situados en las regiones atlántica y pirenaica.

- Centro: parques urbanos de Pamplona/ Iruña, en la región mediterránea.

- Sur de Navarra: formaciones de ribera asociadas a la presencia de grandes ríos (Ebro, Arga, Aragón) y encinares, en la región mediterránea.

\section{Material Y MÉtodos}

Entre septiembre y noviembre de 2012 se han revisado 405 cajas-refugio, que llevaban entre 1 y 3 años sin ser revisadas. Todas se encuentran entre 4 y $7 \mathrm{~m}$ de altura. La inspección se ha realizado accediendo a las cajas mediante una escalera y abriéndolas. Las cajas se encuentran en 11 zonas de Navarra (Fig. 1) y son de 7 modelos distintos: 5 de la marca Schwegler (1FD, 1FF, $1 \mathrm{FW}, 2 \mathrm{FN}, 2 \mathrm{~F}$ de doble pared), un modelo artesano de gran tamaño (Fig. 2) y uno formado por dos tejas superpuestas (Fig. 3). Se han identificado y contado los murciélagos presentes en el interior de cada caja y se han anotado los hallazgos de otros animales o rastro de ellos, prestando especial atención a la presencia de guano de murciélagos. Es preciso matizar que las cajas que carecen de base (modelo 1FF) no suelen almacenar guano, aunque sean ocupadas por murciélagos; por ello, algunas de estas cajas han podido ser ocupadas sin que quede rastro de ello.

Tras la revisión, todas las cajas han sido limpiadas y recolocadas.La mayor parte de las cajas revisadas (61\%) se encuentran en espacios naturales protegidos. 


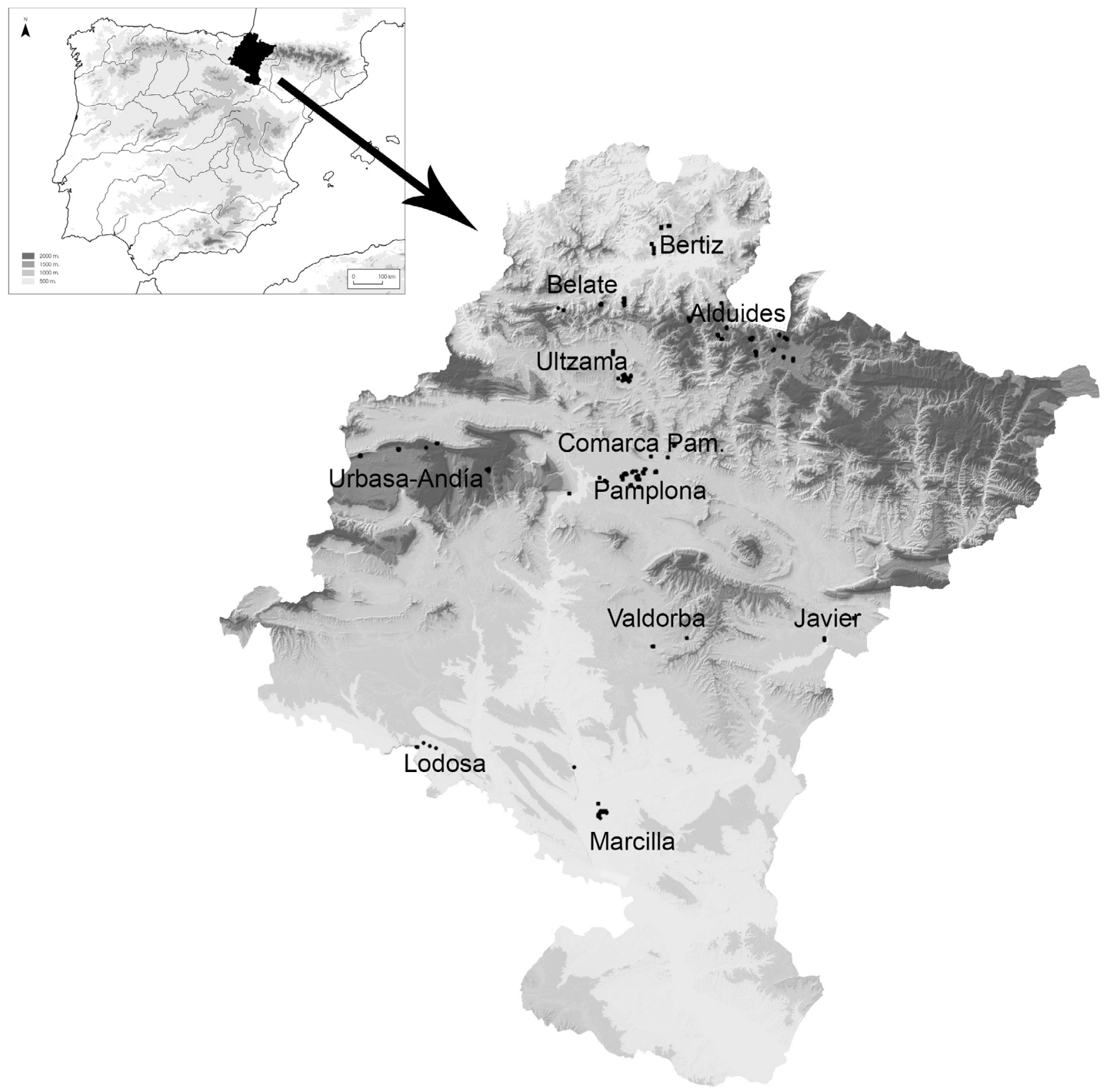

Fig. 1 Cajas-refugio colocadas en Navarra.

Se ha estudiado la ocupación de las cajas en función de las especies presentes, los modelos utilizados, la orientación, los bosques en que se encuentran y su estructura.

\section{Resultados}

Se han encontrado signos de ocupación (guano o murciélagos) en 241 cajas (60\% del total); 107 de ellas (26\%) contenían murciélagos en el momento de la revisión. 47 cajas (12\%) contenían cúmulos de guano tapando todo el fondo. La mayor ocupación y el mayor número de murciélagos se han encontrado en Marcilla, donde se ocupó al menos el $78 \%$ de las cajas, con un promedio de 3,8 individuos por caja (tabla 2).

Se han contabilizado 345 ejemplares que pertenecían a 10 especies (tabla 3). Destaca el murciélago de Cabrera, Pipistrellus pygmaeus, con 247 individuos ( $72 \%$ del total).

Algunas especies son muy dominantes en determinados emplazamientos: en la Comarca de Pamplona, Lodosa y Marcilla todos los murciélagos hallados eran P. pygmaeus; en los parques de Pamplona, el $88 \%$ eran nóctulos medianos, 
Nyctalus noctula; en el robledal de Ultzama, el $83 \%$ eran nóctulos pequeños, Nyctalus leisleri.

Al margen de las 4 cajas de modelos artesanos, todas ellas habitadas, el modelo con mayor ocupación es el 2Fdp (73\%) seguido del 2FN (65\%). Si se tiene en cuenta únicamente la presencia de murciélagos (no de guano), los modelos más ocupados son el $1 \mathrm{FF}(28,5 \%)$ y el 2Fdp (28,0\%), aunque las diferencias observadas no resultan significativas $\left(\mathrm{X}^{2}=3,972 ; \mathrm{p}=0,441\right)$. Esos dos modelos son también los que mayor número de murciélagos presentan por caja: $1,0 \mathrm{y}$ 0,9 respectivamente (tabla 4 ).

Se observa preferencia por esos dos modelos en 3 especies: $P$. pipistrellus, $P$. pygmaeus y $N$. leisleri habitan dichas cajas en mayor porcentaje que las demás $(71 \%, 87 \%$ y $83 \%$ de las cajas ocupadas por cada especie). Por el contrario, el nóctulo mediano, $N$. noctula y el grande, $N$. lasiopterus, se encuentran con más frecuencia en el modelo 2FN ( $88 \%$ y $67 \%$ respectivamente). Las demás especies se han encontrado de forma esporádica.

La mayor ocupación de las cajas se ha producido en platanares $(86 \%)$, jardines con arbolado diverso $(77 \%)$, sotos $(66 \%)$, robledales $(62 \%)$ y hayedos $(54 \%)$, no hallándose diferencias significativas entre ellos $\left(X^{2}=3,442 ; p=0,487\right)$. En los demás bosques, el número de cajas colocadas ha sido muy reducido (entre 3 y 11) y no es posible apreciar tendencias.

Tabla 1. Número y modelos de cajas revisadas en cada localidad (2FdP: modelo 2F de doble pared; otros: modelos variados, artesanos)

\begin{tabular}{|c|c|c|c|c|c|c|c|}
\hline Modelos & 1FD & $1 F W$ & $2 \mathrm{FN}$ & 1FF & 2Fdp & Otros & Total \\
\hline Alduides & & 3 & 27 & 34 & 33 & & 97 \\
\hline Belate & 7 & 3 & 1 & 21 & 9 & 1 & 42 \\
\hline Bertiz & 2 & 3 & 12 & 12 & 26 & & 55 \\
\hline Comarca de Pamplona & 2 & & 9 & 9 & 15 & & 35 \\
\hline Javier & & & 2 & 1 & 7 & & 10 \\
\hline Lodosa & & 1 & & 5 & 3 & & 9 \\
\hline Marcilla & & 3 & & 27 & 22 & 3 & 55 \\
\hline Pamplona & & & 18 & 2 & 2 & & 22 \\
\hline Ultzama & & & 3 & 18 & 15 & & 36 \\
\hline Urbasa-Andia & 1 & 2 & 8 & 8 & 15 & & 34 \\
\hline Valdorba & & & & & 10 & & 10 \\
\hline Total & 12 & 15 & 80 & 137 & 157 & 4 & 405 \\
\hline
\end{tabular}

Tabla 2. Ocupación de las cajas ( $\mathrm{N}$ cajas ocupadas $=\mathrm{N}$ cajas con murciélagos $+\mathrm{N}$ cajas con guano)

\begin{tabular}{lcccc}
\hline & N cajas & N cajas ocupadas & $\begin{array}{c}\text { N cajas con } \\
\text { murciélagos }\end{array}$ & N total murciélagos \\
\hline Alduides & 97 & 59 & 18 & 31 \\
\hline Belate & 42 & 19 & 7 & 8 \\
\hline Bertiz & 55 & 27 & 4 & 8 \\
\hline Comarca de Pamplona & 35 & 15 & 10 & 24 \\
\hline Javier & 10 & 9 & 4 & 6 \\
\hline Lodosa & 9 & 7 & 4 & 14 \\
\hline Marcilla & 55 & 43 & 39 & 209 \\
\hline Pamplona & 22 & 18 & 8 & 16 \\
\hline Ultzama & 36 & 26 & 8 & 12 \\
\hline Urbasa-Andia & 34 & 17 & 4 & 15 \\
\hline Valdorba & 10 & 1 & 1 & 2 \\
\hline Total & 405 & 241 & 107 & 345 \\
\hline
\end{tabular}


Tabla 3. Número de ejemplares de cada especie encontrado en cada zona.

\begin{tabular}{|c|c|c|c|c|c|c|c|c|c|c|c|}
\hline & $\begin{array}{c}\text { B. } \\
\text { barb }\end{array}$ & $\begin{array}{c}\text { M. } \\
\text { daub }\end{array}$ & $\begin{array}{c}\text { M. } \\
\text { myst }\end{array}$ & $\begin{array}{l}N . \\
\text { lasi }\end{array}$ & $\begin{array}{l}N . \\
\text { leis }\end{array}$ & $\begin{array}{c}N . \\
\text { noct }\end{array}$ & $\begin{array}{c}P . \\
\text { auri }\end{array}$ & $\begin{array}{c}P . \\
\text { kuhl }\end{array}$ & $\begin{array}{c}P . \\
\text { pipi }\end{array}$ & $\begin{array}{c}P . \\
\text { pygm }\end{array}$ & Total \\
\hline Alduides & & & 1 & 6 & 14 & & & & 10 & & 31 \\
\hline Belate & 1 & & & & 3 & & & & 4 & & 8 \\
\hline Bertiz & & 1 & & & 2 & & & & 5 & & 8 \\
\hline Comarca de Pamplona & & & & & & & & & & 24 & 24 \\
\hline Javier & & & & & 4 & 2 & & & & & 6 \\
\hline Lodosa & & & & & & & & & & 14 & 14 \\
\hline Marcilla & & & & & & & & & & 209 & 209 \\
\hline Pamplona & & & & & 2 & 14 & & & & & 16 \\
\hline Ultzama & & & & & 10 & & & 2 & & & 12 \\
\hline Urbasa-A. & & & & & 1 & & 7 & & 7 & & 15 \\
\hline Valdorba & & & & & & & & & 2 & & 2 \\
\hline Total & 1 & 1 & 1 & 6 & 36 & 16 & 7 & 2 & 28 & 247 & 345 \\
\hline
\end{tabular}

Tabla 4. Número de cajas utilizadas por cada especie y modelo, y total de ejemplares encontrado por especie y modelo de caja.

\begin{tabular}{|c|c|c|c|c|c|c|c|c|}
\hline & 1FD & $1 F W$ & 2FN & $1 F F$ & 2Fdp & Otros & Cajas con murciélagos & Total murciélagos \\
\hline B. barbastellus & & & & & & 1 & 1 & 1 \\
\hline M. daubentonii & & & & & 1 & & 1 & 1 \\
\hline M. mystacinus & & & & 1 & & & 1 & 1 \\
\hline N. lasiopterus & & & 2 & 1 & & & 3 & 6 \\
\hline N. leisleri & & & 4 & 8 & 12 & & 24 & 36 \\
\hline N. noctula & & & 7 & 1 & & & 8 & 16 \\
\hline P. auritus & & & & & 1 & & 1 & 7 \\
\hline P. kuhlii & & & & 1 & & & 1 & 2 \\
\hline P. pipistrellus & 1 & 2 & 1 & 5 & 5 & & 14 & 28 \\
\hline P.pygmaeus & & 1 & 3 & 22 & 24 & 3 & 53 & 247 \\
\hline $\mathbf{N}$ cajas con murciélagos & 1 & 3 & 17 & 39 & 43 & 4 & 107 & \\
\hline N murciélagos & 1 & 12 & 37 & 138 & 136 & 21 & & 345 \\
\hline $\mathbf{N}$ especies & 1 & 2 & 5 & 7 & 5 & 2 & 10 & 10 \\
\hline
\end{tabular}

Tabla 5. Otros animales y rastros encontrados en las cajas, que dificultaban o impedían su ocupación por murciélagos.

\begin{tabular}{lcccccc}
\hline & 1FD & 1FH & 2FN & 1FF & 2Fdp & Total \\
\hline Avispas & 1 & 1 & 6 & 2 & 12 & 22 \\
\hline Hormigas & & 1 & & & 12 & 13 \\
\hline Lagartija & & 1 & 3 & 12 & 9 \\
\hline Nido roedor & 1 & 1 & 5 & 2 & 3 & 35 \\
\hline Nido ave & & 16 & & 40 & 3 \\
\hline Parásitos & 2 & 7 & 28 & 7 & 84 \\
\hline Total & & & & & & \\
\hline
\end{tabular}




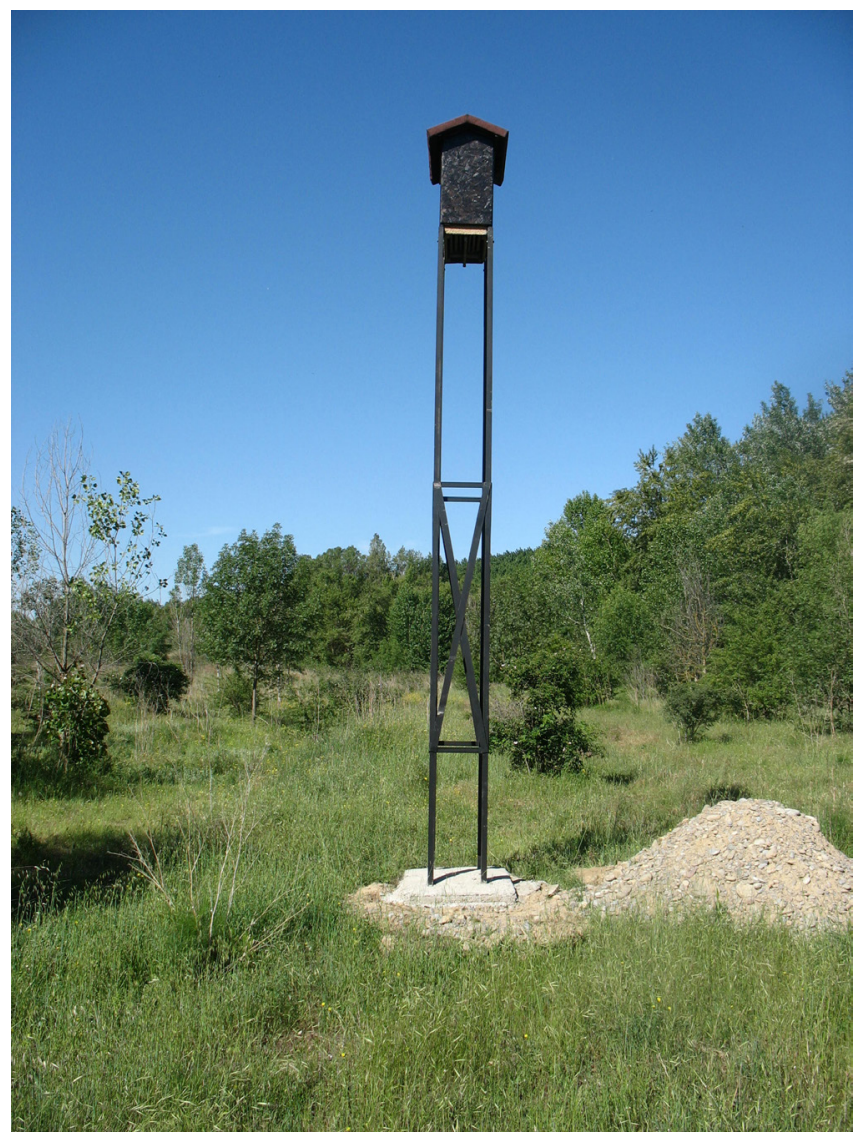

Fig. 2 Caja artesana de gran tamaño colocada en Marcilla. Las tres cajas de este modelo se han ocupado por Pipistrellus pygmaeus.

En los sotos se encuentra la mayor tasa de murciélagos/caja $(2,5)$, aunque en ellos se han encontrado únicamente $P$. pygmaeus; esta especie no se ha hallado en ningún otro bosque. En los platanares se da la segunda mayor tasa $(0,6$ individuos/caja) y en ellos se ha encontrado el $75 \%$ de los ejemplares de $N$. noctula. Los demás bosques muestran tasas de ocupación de 0,3 ind./ caja (jardines, hayedos y robledales) o inferiores.

La ocupación de las cajas es mayor cuanto más abierta es la estructura del bosque y viceversa; las diferencias encontradas no llegan a ser significativas al considerar las cajas ocupadas $\left(X^{2}=7,09 ; p=0,069\right)$, aunque sí resultan significativas si consideramos el número de murciélagos por caja $\left(X^{2}=136,64 ; p<0,001\right)$ (Fig. 4).

Las cajas más ocupadas son las orientadas al S (71\%) y al NO (66\%), mientras que las menos ocupadas son las orientadas al NE (53\%) $\mathrm{y}$ al O (56\%); estas pequeñas diferencias no son significativas $\left(\mathrm{X}^{2}=0,92 ; \mathrm{p}=0,99\right)$. Sin embargo, al comparar el número de murciélagos encontrado en las cajas en función de su orientación, las

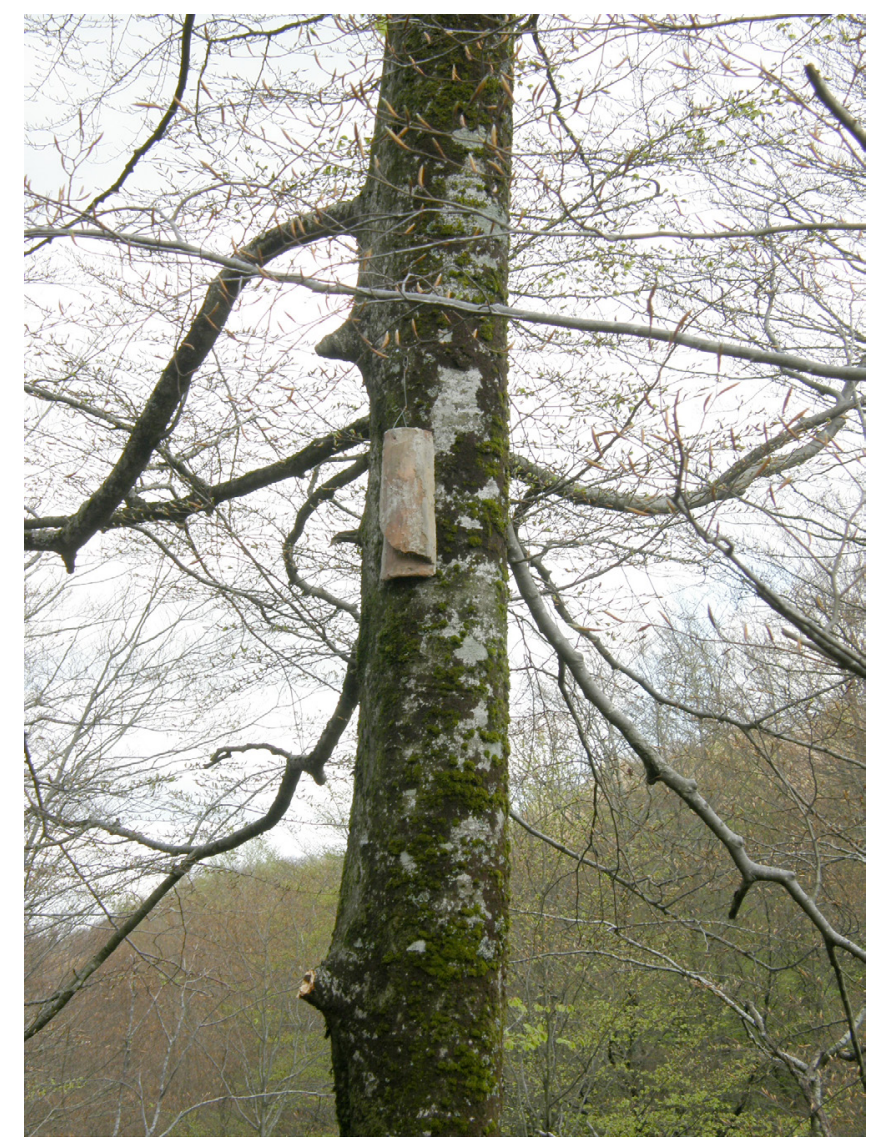

Fig. 3 Refugio formado por dos tejas, en Belate. En el único refugio colocado de este modelo se encontró un macho de Barbastella barbastellus.

diferencias sí resultan significativas $\left(\mathrm{X}^{2}=15,53\right.$; $\mathrm{p}=0,03)$, siendo más usadas las cajas orientadas al NO (1,38 ind./caja), SO (1,03) y S (1,00), mientras que las orientadas al NE presenta menor número de individuos por caja $(0,48)$.

El $21 \%$ de las cajas revisadas estaban ocupadas por otros seres vivos o rastros de ellos que impedían o dificultaban la ocupación de las mismas por murciélagos. Destacan los nidos de aves (9\%) y los avisperos (5\%). Únicamente dos cajas con avispas activas albergaban murciélagos (P. pygmaeus). Ninguna de las tres cajas halladas con abundantes parásitos (ácaros), presentaba murciélagos, aunque todas contenían guano. Ver tabla 5 .

\section{DisCUSIÓN}

La instalación de cajas-refugio es una medida ampliamente utilizada para favorecer el asentamiento de murciélagos arborícolas o fisurícolas (e.g., Taake \& Hildenhagen 1989, Altringham 1998, Paz et al. 2000) y otras especies de mamíferos amenazadas (Lindenmayer et al. 


\section{Ocupación de las cajas y estructura del bosque}

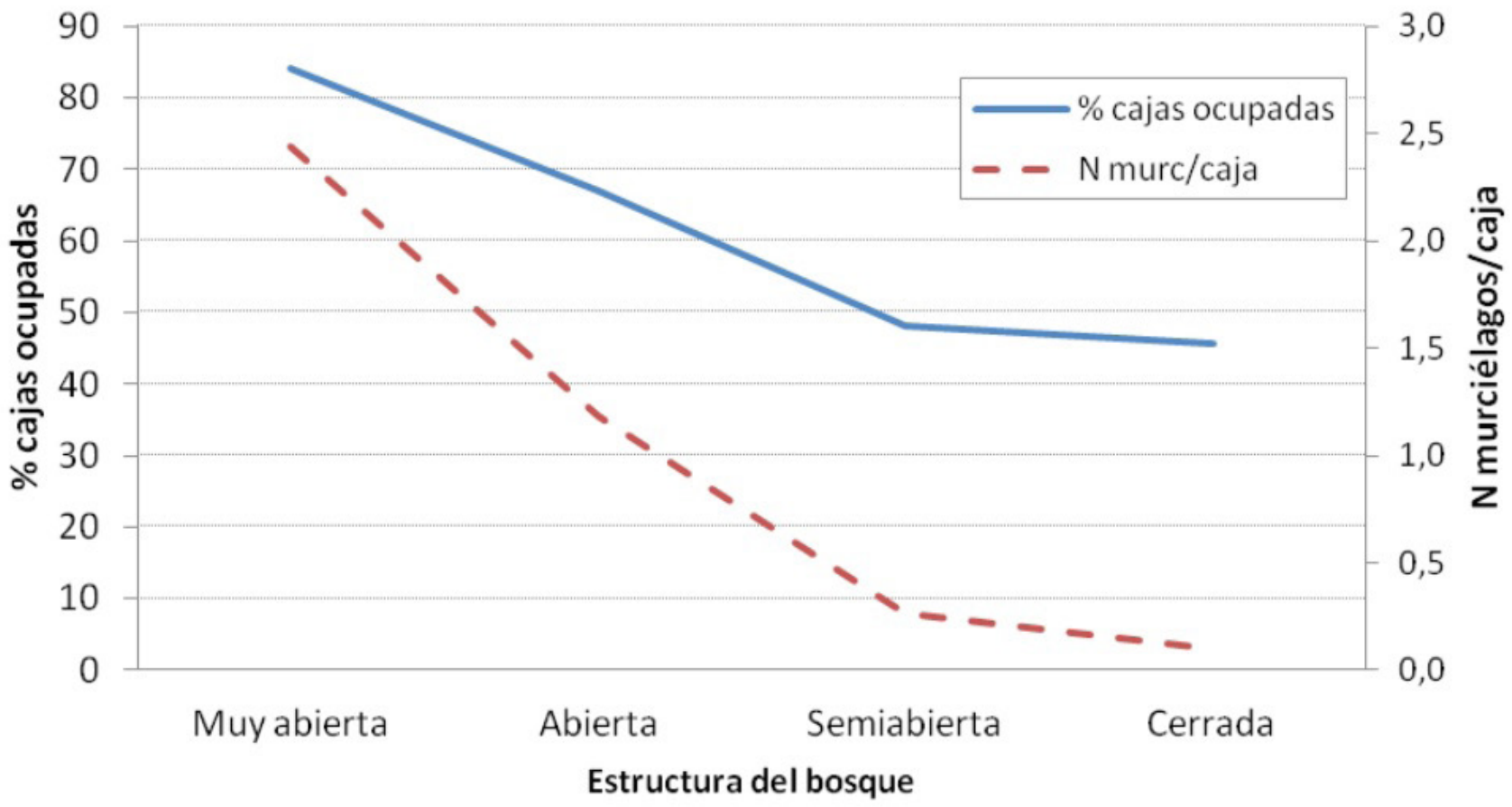

Fig. 4. Ocupación de las cajas en función de la estructura forestal (cerrada: dosel tapando el cielo; semiabierta: dosel con huecos; abierta: borde de bosque, hasta $180^{\circ}$ de su entorno libre de arbolado; muy abierta: árbol aislado o descubierto en más de la mitad de su contorno).

2009). Sin embargo, la eficacia de medida es cuestionada por algunos autores, al temer que dichas cajas provoquen un efecto atrayente sobre especies oportunistas y abundantes, en detrimento de otras más escasas y exigentes (Boye \& Dietz 2005), por lo que se recomienda dirigir esta actuación a zonas con déficit de refugios naturales. En cualquier caso, las cajas pueden paliar la escasez o insuficiencia de refugios naturales en determinadas áreas, debido a una gestión forestal presente o pasada, pero no deben sustituir la necesaria presencia de árboles con oquedades naturales (Mitchell-Jones $\&$ McLeish 2004), que debe ser una prioridad de una gestión forestal sostenible (Gibbons et al. 2010). Aunque las cajas colocadas en Navarra se encuentran principalmente en espacios protegidos, han sido instaladas mayoritariamente en bosques sometidos a explotación forestal y por tanto con escasez de refugios naturales. En este sentido la generación natural de oquedades en los bosques templados es un fenómeno asociado a edades avanzadas del arbolado (Sandström 1992) y por tanto, los refugios pueden ser una herramienta para paliar este déficit a corto-medio plazo.Por otro lado, las cajas-refugio pueden constituir un valioso instrumento para conocer las especies presentes en una zona, o para valorar la evolución de las poblaciones que las ocupan (Boye \& Dietz 2005).

La revisión ha permitido comprobar la mayor eficacia de las cajas de cemento-madera (Gerell 1985) respecto a las de madera así como su mayor durabilidad (Stebbings \& Walsh 1991): las más antiguas se encontraban en buenas condiciones y aptas para su uso, tras 12 años a la intemperie.

En conjunto, las cajas revisadas han tenido una ocupación notable (60\%) y diversa (10 especies de quirópteros), aunque la heterogeneidad de los resultados (entre el 10 y el $90 \%$ de ocupación según zonas) revela claras diferencias en la aceptación de estos refugios. Conviene matizar que en la zona con menor ocupación (Valdorba $10 \%$ ), las cajas fueron colocadas por personal desconocedor de los murciélagos, y varias cajas se encontraban en zonas de espesura, difícilmente accesibles para estos mamíferos; ello evidencia la necesidad de contar con técnicos especializados en quirópteros durante la realización de proyectos que incluyan la colocación y revisión de cajasrefugio. Cabe achacar la elevada diversidad de especies encontrada a las peculiares condiciones 


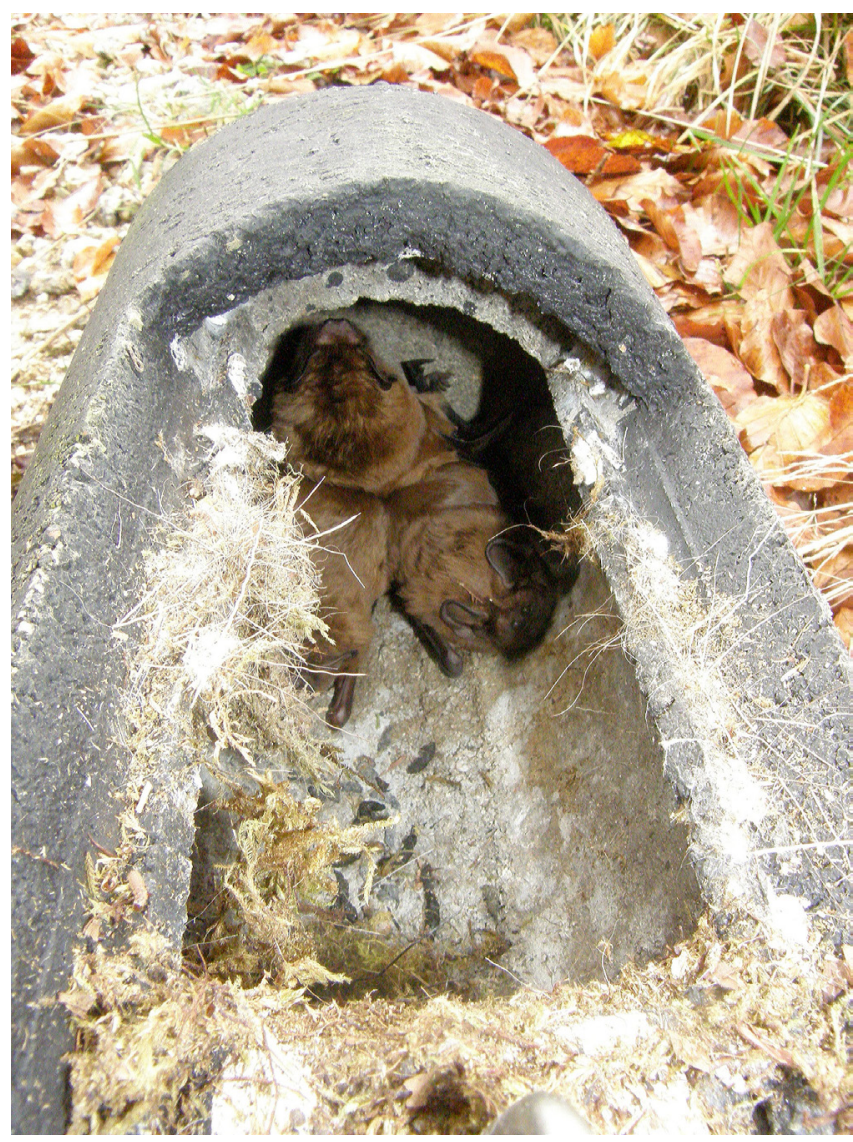

Fig. 5 Harén de Nyctalus lasiopterus descansando en una caja Schwegler 2FN (1 macho y 2 hembras).

biogeográficas de la zona de estudio, donde confluyen las tres regiones bioclimáticas de la península Ibérica en una zona de paso migratorio de murciélagos y a la diversidad de hábitats en los que se han instalado estos refugios.

Aunque a nivel global no se han apreciado diferencias significativas en la ocupación de diferentes modelos de cajas, se ha observado la preferencia de determinados modelos por parte de diferentes especies. Por ello, el conocimiento previo de las especies existentes en una zona puede ayudar a elegir los modelos de cajas más adecuados y en definitiva, mejorar la eficacia de esta medida. También es importante considerar que los distintos modelos de cajas tienen diferente coste económico, por lo que una elección adecuada puede también maximizar la relación beneficiocoste de la actuación.

Las observaciones realizadas en relación a la estructura del bosque, aconsejan la colocación de cajas en zonas abiertas o muy abiertas, lo cual está en consonancia con las recomendaciones de algunos autores por el uso de postes o árboles en zonas despejadas y soleadas (Tuttle \& Hensley 1993, Lourenço \& Palmeirim 2003). En el presente trabajo se aprecia una mayor presencia de murciélagos en cajas orientadas hacia el $\mathrm{O}$ y el $\mathrm{S}$, aunque si se atiende únicamente a los rastros encontrados, no se observan diferencias. En el centro de la península Ibérica, Paz et al. (2000) tampoco encuentran diferencias. En Centro Europa los murciélagos prefieren cajas orientadas al SO o al S, prestando poca atención a las orientadas hacia el E (Boye \& Dietz 2005). A la vista de estas observaciones, cabe sospechar que la preferencia por determinadas orientaciones varíe en función de otros factores más relevantes, como la latitud o la estacionalidad. En este sentido cabe recordar que la presencia de excrementos puede deberse a ocupaciones de las cajas en cualquier época del año, mientras que en el presente trabajo, los murciélagos se han hallado únicamente en otoño. En este período existe una gran variación térmica entre el día y la noche, por lo que la orientación de las cajas puede ser entonces relevante.

La notable dominancia de especies concretas en determinados lugares muestra una distribución contagiosa de sus efectivos, que puede estar ligada al comportamiento propio de algunas especies como los nóctulos medianos, que forman "leks" en otoño (Sluiter \& van Heerdt 1986), comportamiento observado en Pamplona (Alcalde 2008) y/o a la selección de determinados hábitats. En el caso de los nóctulos, destaca el hallazgo en las cajas de machos solitarios en celo y harenes de las tres especies peninsulares Aunque éstas se encuentran en Navarra durante todo el año, machos y hembras parecen distribuirse por diferentes áreas durante el verano, debido probablemente a sus diferentes necesidades energéticas (Barclay \& Kurta 2007). En la región, sólo se han observado hembras de las tres especies de nóctulos entre finales de agosto y mediados de marzo, lo que presupone

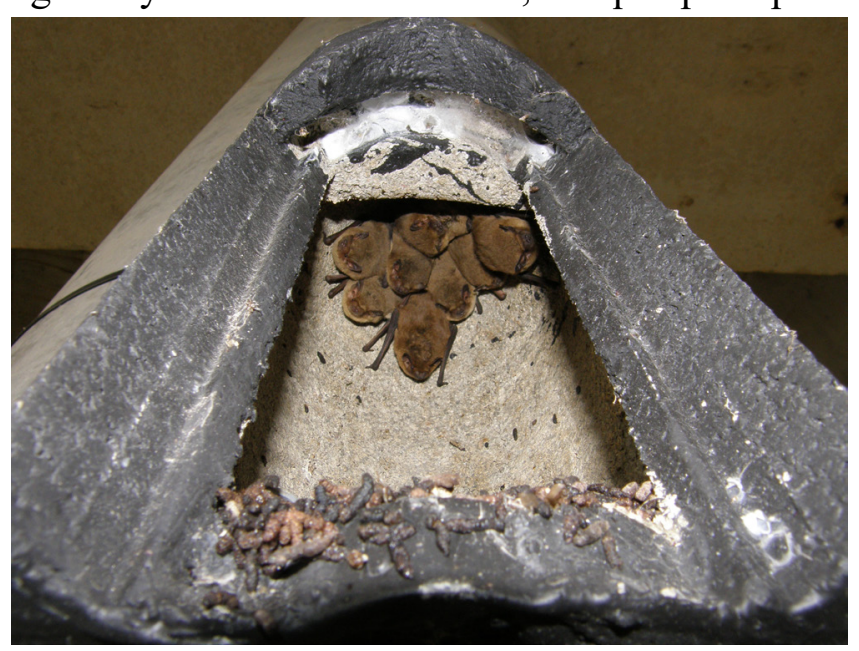

Fig. 6 Harén de Pipistrellus pygmaeus en una caja Schwegler 2F de doble pared (1 macho y 10 hembras). 
un comportamiento migrador (Ibáñez et al. 2009) constatado en la Península Ibérica para el nóctulo pequeño (Ohlendorf et al. 2000, Wohlgemuth et al. 2004, Alcalde et al. en prensa). En este sentido cabe destacar la posición geográfica de Navarra, situada en el trayecto de los ejemplares que migran entre Centro Europa y la Península Ibérica: durante este recorrido, las cajas pueden tener un especial valor como refugios accesibles y fáciles de localizar. Además, en ocasiones se ha comprobado la ocupación de las cajas por nóctulos pequeños y medianos para la hibernación (Alcalde 2008, Alcalde et al. en prensa). En ningún caso se encontraron nóctulos muertos en las cajas, como ha ocurrido en Alemania, donde se atribuye el hecho a una posible congelación (Boye \& Dietz 2005) o a la falta de oxígeno por exceso de ejemplares (Arthur \& Lemaire 2009). N. noctula, además, parece mostrar preferencia por los platanares, donde se encuentra la mayoría de sus ejemplares, al igual que se ha observado en Francia (Arthur \& Lemaire 2009).

La dominancia de P. pygmaeus en Marcilla, Lodosa y Comarca de Pamplona, cuyas cajas se sitúan en sotos de zonas rurales, y el notable éxito de las mismas, parecen deberse su preferencia por los hábitats acuáticos; estas observaciones son coincidentes con las realizadas por Flaquer et al. (2005) en cajas del Delta del Ebro, donde la especie es muy abundante y llega a ocupar hasta el $95,6 \%$ de las cajas.

Llama la atención el hallazgo de un solo ejemplar de B. barbastellus refugiado en el único refugio formado por dos tejas superpuestas. Esta especie es relativamente frecuente en la mitad septentrional de la región, aunque parece evitar los diferentes modelos cajas, salvo el modelo mencionado, que simula las grietas existentes bajo la corteza, su refugio habitual (Meschede \& Keller 2003). Este modelo, de bajo coste y fácil construcción, podría ser muy adecuado para dicha especie en zonas con escasez de refugios.

El hallazgo de un $21 \%$ de cajas ocupadas por otros organismos que pueden dificultar o impedir el acceso a los murciélagos, así como un $12 \%$ de cajas con abundante guano, revelan la necesidad de realizar limpiezas periódicas de las mismas; se ha observado que un exceso de guano puede provocar el abandono de las mismas (Heise \& Blohm 1998) y los nidos de aves pueden llegar a taponar la entrada de las cajas, impidiendo su ocupación (Alcalde 2008); además, algunos paseriformes pueden atacar y devorar murciélagos (Estók et al. 2010). Por ello, y a la vista de estos resultados, se recomienda una revisión y limpieza de estos refugios al menos cada 2-3 años.

Por último, cabe mencionar que los datos encontrados en este trabajo se ciñen únicamente a un área geográfica muy concreta, y es posible que en otras zonas, los murciélagos muestren diferentes preferencias o requerimientos; por ello, convendría realizar más experiencias similares que contribuyesen a conocer mejor los factores que influyen en la ocupación de cajas-refugio por diferentes especies de murciélagos y en definitiva a mejorar la eficacia de estas actuaciones.

\section{Agradecimientos}

La revisión de las cajas-refugio se ha realizado gracias a la financiación del Gobierno de Navarra por medio de Gestión Ambiental de Navarra, S.A. y a la Mancomunidad de la Comarca de Pamplona. Además en la revisión han ayudado y colaborado con gran interés Alfonso Senosiain, Inmaculada Siles, Javier Zuazu y Xabi Lopetegi de la Sección de Guarderío del Departamento de Desarrollo Rural, Medio Ambiente y Administración Local del Gobierno de Navarra y numerosos voluntarios de la asociación Alnus, de Marcilla, y Gabriel Fuentes por su colaboración con la construcción de cajas.

\section{REFERENCIAS}

AlCalde, J. T. 2008. El nóctulo mediano en Pamplona. Biodiversidad Urbana de Pamplona. Ayuntamiento de Pamplona, Pamplona.

AlCalde, J. T., IbáÑez, C., Antón, I. \& Nyssen, P. 2012. First case of migration of a Leisler's bat (Nyctalus leisleri) between Spain and Belgium. Le Rhinolophe, en prensa.

Alerstam, T. 1990. Birds Migration. Cambridge University Press, Cambridge.

Arthur, L. \& Lemaire, M. 2009. Les Chauvessouris de France, Belgique, Luxembourg et Suisse. Biotope. Museum National d'Histoire Naturelle, París.

Altringham, J. 1998. Bat houses in British forests. Bats 16: 8-11. 
Barclay, R. M. R. \& Kurta, A. 2007. Ecology and behavior of bats roosting in tree cavities and under bark. En: Lacki M. J., Hayes, J. P. \& Kurta, A. (Eds.) Bats in forests. Conservation and management. The Johns Hopkins University Press, Baltimore.

Boye, P. \& Dietz, M. 2005. Development of good practice guidelines for woodland management for bats. The Bat Conservation Trust. English Nature, Peterborough, 90 pp

ElPhick, J. 1995. The Atlas of Bird Migration. Marshall Editions, London.

EstóK, P., ZsebőK, S. \& SIEMERS, B. M. 2010. Great tits search for, capture, kill and eat hibernating bats. Biology Letters 6: 59-62. DOI: https:// dx.doi.org/10.1098/rsbl.2009.0611

Flaquer, C., Torre, I. \& Ruiz-Jarillo, R. 2006. The value of bat-boxes in the conservation of Pipistrellus pygmaeus in wetland rice paddies. Biological Conservation 128: 223230. DOI: https://dx.doi.org/10.1016/j. biocon.2005.09.030

Gerrell, R. 1985. Tests of boxes for bats. Nyctalus 2: 181-185.

Gibbons, P., McElhinny, C. \& Lindenmayer, D. B. 2010. What strategies are effective for perpetuating structures provided by old trees in harvested forests? A case study on trees with hollows in south-eastern Australia. Forest Ecology and Management 260: 975982. DOI: https://dx.doi.org/10.1016/j. foreco.2010.06.016

Heise, G. \& Blohm, T. 1998. Welche Ansprüche stellt der Abendsegler (Nyctalus noctula) an das Wochenstubenquartier? Nyctalus (N. F.) 6: 471-475.

IbáÑez, C., Guillén, A., Agirre-Mendi, P. T., Juste, J., Schreur, G., Cordero, A., \& PopALisseanu, A. 2009. Sexual segregation in Iberian noctule bats. Journal of Mammalogy 90(1): 235-243. DOI: http://dx.doi. org/10.1644/08-MAMM-A-037.1

Lindenmayer, D. B., Welshb, A., Donnellya, C., Crane, M., Michael, D., Macgregor, C., McBurney, L., Montague-Drake, R. \& GibBons, P. 2009. Are nest boxes a viable alternative source of cavities for hollow-dependent animals? Long-term monitoring of nest box occupancy, pest use and attrition. Biological Conservation 142: 33-42. DOI: https://dx.doi.org/10.1016/j. biocon.2008.09.026

LOURENÇO, S.I.\&PALMEIRIM,J.M.2004.Influence of temperature in roost selection by Pipistrellus pygmaeus (Chiroptera): relevance for the design of bat boxes. Biological Conservation 119: 237-243. DOI: https://dx.doi. org/10.1016/j.biocon.2003.11.006

Meschede, A. \& Keller, K. G. 2003. Écologie et protection des chauves-souris en milieu forestier. Le Rhinolophe 16: 1-248.

Mitchell-Jones, A. J. \& McLeish, A. P. 2004. Bat workers manual ( $3^{\mathrm{a}}$ edición). Joint Nature Conservation Committee, Petterborough.

Ohlendorf, V. B., Hecht, B., Strassburg, D. \& Agirre-Mendi, P. T. 2000. Fernfund eines Kleinabendseglers (Nyctalus leisleri) in Spanien. Nyctalus (N.F.) 7 (3): 239-242.

Paz, Ó. DE, Lucas, J. DE \& ARIAs, J. 2000. Cajas refugio para quirópteros y estudio de la población del murciélago orejudo dorado (Plecotus auritus linneo, 1758) en un área forestal de la provincia. Ecología 14: 259268 .

SANDSTRÖM, U. 1992. Cavities in trees, their occurrence, formation and importance for hole-nesting birds in relation to silvicultural practise. Swedish University of Agricultural Sciences, Uppsala.

Sluiter, J. W. \& van Heerdt, P. F. 1966. Seasonal habits of the noctula bat (Nyctalus noctula). Archives Neerlandaises de Zoologie 16, 423-439.

Stebbings, R. E. \& Walsh, S. T. 1991. Bat boxes. A guide to the History, Function, Construction and Use in the Conservation of Bats. The Robert Stebbings Consultancy Ltd., Peterborough.

TAake, K. H. \& Hildenhagen, U. 1989. Nine years' inspection of different artificial roost for forest- dwelling bats in Northern Westfalia: some results. En: Hanák, V., Horácek, I. \& Gaisler, J. (Eds). European Bat Research 1987. Pp: 487-493. Charles University Press, Praha. 
Tuttle, M. D. \& Hensley, D. L. 1993. The Bat Wohlgemuth, R., Devrient, I., García, A., \& House Builder's Hand- book. Bat Conservation International, Texas.

Hutterer, R. 2004. Long-distance flight of a Lesser noctule (Nyctalus leisleri) after rehabilitation. Myotis 41-42: 69-73. 